\title{
Stable receding horizon control for max-plus-linear systems*
}

I. Necoara, B. De Schutter, T.J.J. van den Boom, and J. Hellendoorn

If you want to cite this report, please use the following reference instead:

I. Necoara, B. De Schutter, T.J.J. van den Boom, and J. Hellendoorn, "Stable receding horizon control for max-plus-linear systems," Proceedings of the 2006 American Control Conference, Minneapolis, Minnesota, pp. 4055-4060, June 2006.

Delft Center for Systems and Control

Delft University of Technology

Mekelweg 2, 2628 CD Delft

The Netherlands

phone: +31-15-278.24.73 (secretary)

URL: https: / / www.dcsc.tudelft.nl

*This report can also be downloaded viahttps://pub.deschutter.info/abs/05_016.html 


\title{
Stable Receding Horizon Control for Max-Plus-Linear Systems
}

\author{
I. Necoara, B. De Schutter, T.J.J. van den Boom and J. Hellendoorn
}

\begin{abstract}
We develop a stabilizing receding horizon control (RHC) scheme for the class of discrete-event systems called max-pus-linear (MPL) systems. MPL systems can be described by models that are "linear" in the max-plus algebra, which has maximization and addition as basic operations. In this paper we extend the concept of positively invariant set from classical system theory to discrete-event MPL systems. We define stability for the class of MPL systems in the sense of Lyapunov. For a particular convex piecewise affine cost function and linear input-state constraints the RHC optimization problem can be recast as a linear program. Using a dual-mode approach we are able to prove exponential stability of the RHC scheme. We derive also a constrained time-optimal controller by solving a sequence of parametric linear programs.
\end{abstract}

\section{INTRODUCTION}

In the last decades Receding Horizon Control (RHC) or Model Predictive Control (MPC) [1], [2] has gained wide acceptance in the process industry. An important advantage of RHC is that the use of a finite horizon allows the inclusion of constraints on the inputs and states. Recently, the RHC approach was extended to a class of discrete-event systems (DES) called max-plus-linear (MPL) systems [3]. MPL systems are linear in the max-plus algebra [4] and they usually arise in the context of manufacturing systems, telecommunication networks, railway networks, parallel computing etc. Several authors have already developed methods to compute optimal controllers for MPL systems [3], [5]-[8]. The main advantage of the RHC scheme presented in this paper is that it allows to include linear constraints on inputs and states and the RHC controller guarantees a priori stability of the closed-loop system.

We start the paper with an introduction of the main concepts from max-plus algebra. We introduce stability in the sense of Lyapunov for the class of MPL systems, using similar concepts as in [9]. In Section II we take into account constraints on input and states. We define the concept of positively invariant (PI) set for the class of MPL systems. We prove that, under some mild conditions, the PI set is a polyhedron. For a particular convex piecewise affine cost function, we prove that the MPL-RHC optimization problem can be recast as a linear program (LP). Using a dual-mode approach [1] we prove that the RHC controller stabilizes in the sense of Lyapunov the MPL system. In Section III we derive a time-optimal controller using parametric linear programming. We conclude with an example.

I. Necoara, B. De Schutter, T.J.J. van den Boom and J. Hellendoorn are with Delft Center for Systems and Control, Delft University of Technology, Mekelweg 2, 2628 CD Delft, The Netherlands \{i.necoara,b. deschutter,t.j.j.vandenboom, j.hellendoorn\}@dcsc.tudelft.nl

\section{A. Max-Plus Algebra}

Define $\varepsilon:=-\infty$ and $\mathbb{R}_{\varepsilon}:=\mathbb{R} \cup\{\varepsilon\}$. The max-plusalgebraic (MPA) addition $(\oplus)$ and multiplication $(\otimes)$ are defined as [4]: $x \oplus y:=\max \{x, y\}, x \otimes y:=x+y$, for $x, y \in$ $\mathbb{R}_{\varepsilon}$. For matrices $A, B \in \mathbb{R}_{\varepsilon}^{m \times n}$ and $C \in \mathbb{R}_{\varepsilon}^{n \times p}$ one can extend the definition as follows: $(A \oplus B)_{i j}:=A_{i j} \oplus B_{i j},(A \otimes C)_{i j}:=$ $\bigoplus_{k=1}^{n} A_{i k} \otimes C_{k j}, \forall i, j$. The matrix $\mathcal{E}$ denotes the MPA zero matrix of appropriate dimension: $\mathcal{E}_{i j}:=\varepsilon, \forall i, j$ and $E_{n}$ is the $n \times n$ MPA identity matrix: $\left(E_{n}\right)_{i i}:=0, \forall i$ and $\left(E_{n}\right)_{i j}:=\varepsilon$, $\forall i, j$ with $i \neq j$. For any matrix $A \in \mathbb{R}_{\varepsilon}^{n \times n}$, let $A^{\otimes^{k}}:=$ $A \otimes A \otimes \cdots \otimes A$ (i.e. the $k$ th MPA power of $A$ ) and define $A^{*}$, whenever it exists, by $A^{*}:=E_{n} \oplus A \oplus \cdots \oplus A^{\otimes^{k}} \oplus \cdots$ Given a vector $x \in \mathbb{R}_{\mathcal{\varepsilon}}^{n}$ we denote with $\|x\|_{\oplus}:=\max \left\{x_{1} \cdots x_{n}\right\}$. For a positive integer $n$, we denote with $\underline{n}:=\{1,2, \cdots, n\}$. A matrix $\Gamma \in \mathbb{R}_{\varepsilon}^{n \times m}$ is row-finite if for any row $i \in \underline{n}, \max _{j \in \underline{m}} \Gamma_{i j}>\varepsilon$; column-finite is similarly defined.

We denote with $x \oplus^{\prime} y:=\min \{x, y\}$ and $x \otimes^{\prime} y:=x+y$ (the operations $\otimes$ and $\otimes^{\prime}$ differ only in that $(-\infty) \otimes(+\infty):=-\infty$, while $\left.(-\infty) \otimes^{\prime}(+\infty):=+\infty\right)$. The matrix multiplication and addition for $\left(\oplus^{\prime}, \otimes^{\prime}\right)$ are defined similarly as for $(\oplus, \otimes)$. It can be shown that for any matrices $A, B$ and any vectors $x, y$ of appropriate dimensions over $\mathbb{R}_{\varepsilon}$ we have [10]:

$$
\begin{aligned}
& A \otimes^{\prime}(B \otimes x) \geq\left(A \otimes^{\prime} B\right) \otimes x,\left(\left(-A^{T}\right) \otimes^{\prime} A\right) \otimes x \geq x, \\
& x \leq y \Rightarrow A \otimes x \leq A \otimes y \text { and } A \otimes^{\prime} x \leq A \otimes^{\prime} y .
\end{aligned}
$$

Lemma 1.1: [4] (i) The inequality $A \otimes x \leq b$ has the largest solution given by $x_{\mathrm{opt}}=\left(-A^{T}\right) \otimes^{\prime} b=-\left(A^{T} \otimes(-b)\right)$ (by the largest solution we mean that for all $x$ satisfying $A \otimes x \leq b$ we have $x \leq x_{\text {opt }}$ ).

(ii) The equation $x=A \otimes x \oplus b$ has a solution $x=A^{*} \otimes b$. If $A_{i j}<0$ for all $i, j$, then the solution is unique.

\section{B. Max-Plus-Linear Systems}

DES with only synchronization and no concurrency can be modeled by an MPA model of the following form [4]:

$$
\left\{\begin{array}{l}
x_{\text {sys }}(k)=A_{\text {sys }} \otimes x_{\text {sys }}(k-1) \oplus B_{\text {sys }} \otimes u_{\text {sys }}(k), \\
y_{\text {sys }}(k)=C_{\text {sys }} \otimes x_{\text {sys }}(k)
\end{array}\right.
$$

where $x_{\text {sys }}(k) \in \mathbb{R}_{\varepsilon}^{n}$ represents the state, $u_{\text {sys }}(k) \in \mathbb{R}_{\varepsilon}^{m}$ is the input, $y_{\mathrm{sys}}(k) \in \mathbb{R}_{\varepsilon}^{p}$ is the output and where $A_{\text {sys }} \in \mathbb{R}_{\varepsilon}^{n \times n}$, $B_{\text {sys }} \in \mathbb{R}_{\mathcal{E}}^{n \times m}, C_{\text {sys }} \in \mathbb{R}_{\mathcal{\varepsilon}}^{p \times n}$ are the system matrices ${ }^{1}$. Since the states and the inputs represent times, typical constraints

\footnotetext{
${ }^{1}$ We may assume without loss of generality that $B_{\text {sys }}$ is column-finite and $C_{\text {sys }}$ is row-finite.
} 
for MPL systems are (see [8] for more details):

$$
\left\{\begin{array}{l}
u_{\text {sys }}(k+1)-u_{\text {sys }}(k) \geq 0, \\
H_{\text {sys }} x_{\text {sys }}(k)+G_{\text {sys }} u_{\text {sys }}(k) \leq h_{\text {sys }}(k)
\end{array}\right.
$$

Let $\lambda^{*}$ be the largest MPA eigenvalue of $A_{\text {sys }}$ (see [4] for an appropriate definition). We consider a reference signal that the output should track of the form:

$$
r_{\text {sys }}(k)=y_{\text {sys }, \mathrm{t}}+k \rho \text {. }
$$

Since through the term $B_{\text {sys }} \otimes u_{\text {sys }}$ it is only possible to create delays in the starting times of activities, we should choose the growth rate of the due dates such that is larger than the growth rate of the system, i.e. $\rho \geq \lambda^{*}$. If $\lambda^{*}>\varepsilon$ (in practical applications we even have $\lambda^{*} \geq 0$ ) then there exists an MPA invertible matrix $P \in \mathbb{R}_{\varepsilon}^{n \times n}$ such that the matrix $\bar{A}=P^{\otimes^{-1}} \otimes A_{\text {sys }} \otimes P$ satisfies $\bar{A}_{i j} \leq \lambda^{*}$, for all $i, j \in \underline{n}[11]$ $\left(P^{\otimes}\right.$ denotes the MPA inverse of $P$ ). We make the following change of coordinates: $\bar{x}(k)=P^{\otimes^{-1}} \otimes x_{\text {sys }}(k)$. We denote with $\bar{B}=P^{\otimes^{-1}} \otimes B_{\text {sys }}, \bar{C}=C_{\text {sys }} \otimes P$ and $\bar{y}(k)=y_{\text {sys }}(k), \bar{u}(k)=$ $u_{\text {sys }}(k)$. In the new coordinates the system (2) becomes:

$$
\bar{x}(k)=\bar{A} \otimes \bar{x}(k-1) \oplus \bar{B} \otimes \bar{u}(k), \bar{y}(k)=\bar{C} \otimes \bar{x}(k)
$$

We now consider the normalized system: $x(k)=\bar{x}(k)-$ $\rho k, u(k)=\bar{u}(k)-\rho k, y(k)=\bar{y}(k)-\rho k, A=\bar{A}-\rho$ (i.e. by subtracting in the conventional algebra all entries of $\bar{x}, \bar{u}, \bar{y}$ and of $\bar{A}$ by $\rho k$ and $\rho$, respectively) and $B=\bar{B}, C=\bar{C}$. The normalized system can be written as:

$$
\begin{aligned}
& x(k)=A \otimes x(k-1) \oplus B \otimes u(k) \\
& y(k)=C \otimes x(k) .
\end{aligned}
$$

We assume that in the new coordinates, the constraint (3) becomes:

$$
u(k+1)-u(k) \geq-\rho, H x(k)+G u(k) \leq h .
$$

The following assumption will be used throughout the paper:

Assumption A: We consider that $\rho>\lambda^{*} \geq 0$, the system is controllable and observable ${ }^{2}$ and $H \geq 0$ in (6).

The conditions from Assumption $\mathbf{A}$ are quite weak and are usually met in applications. Note that $\rho$ can be chosen arbitrarily close to $\lambda^{*}$. From Assumption $\mathbf{A}$ it follows that $A_{i j}<0$, for all $i, j \in \underline{n}$. In the new coordinates the output should be regulated to the desired target $y_{\mathrm{t}}:=y_{\mathrm{sys}, \mathrm{t}}$.

Since $A_{i j}<0$ for all $i, j \in \underline{n}, A^{*}=E_{n} \oplus A \oplus \cdots \oplus A^{\otimes^{n-1}}$ (see [4]). Note that for any finite vector $u$ there exists a state equilibrium $x$ (i.e. $x=A \otimes x \oplus B \otimes u$ ), given by $x=A^{*} \otimes B \otimes u$. Note that $x$ is unique (according to Lemma 1.1 (ii)) and finite (due to controllability assumption). We associate to $y_{\mathrm{t}}$ the largest ${ }^{3}$ equilibrium pair $\left(x_{\mathrm{e}}, u_{\mathrm{e}}\right)$ satisfying $C \otimes x_{\mathrm{e}} \leq y_{\mathrm{t}}$. From the previous discussion and taking into account that the system is observable it follows that $\left(x_{\mathrm{e}}, u_{\mathrm{e}}\right)$ is unique, finite and given by (see also [8]):

$$
u_{\mathrm{e}}=\left(-\left(C \otimes A^{*} \otimes B\right)\right)^{T} \otimes^{\prime} y_{\mathrm{t}}, x_{\mathrm{e}}=A^{*} \otimes B \otimes u_{\mathrm{e}}
$$

\footnotetext{
${ }^{2}$ See [4] for appropriate definitions for observability and controllability. ${ }^{3} \mathrm{By}$ the largest we mean that any other feasible equilibrium pair $(x, u)$ satisfies $x \leq x_{\mathrm{e}}, u \leq u_{\mathrm{e}}$.
}

Throughout the paper $\|\cdot\|_{\infty}$ denotes the $\infty$-norm $\left(\|x\|_{\infty}:=\right.$ $\left.\max _{i \in \underline{n}}\left|x_{i}\right|\right)$.

\section{Lyapunov stability for MPL systems}

In this section we adopt the formulation developed in [9] to the study of stability of MPL systems. Let $d_{\infty}$ denote the metric on $\mathbb{R}^{n}$ induced by $\infty$-norm. Given a set $\mathscr{O} \subset \mathbb{R}^{n}$ then $d_{\infty}\left(x_{0}, \mathscr{O}\right)=\min _{x \in \mathscr{O}}\left\|x_{0}-x\right\|_{\infty}$ denotes the distance from a point $x_{0}$ to the set $\mathscr{O}$. An $r$-neighborhood of a set $\mathscr{O}$ is defined as the set $\mathscr{N}(\mathscr{O}, r)=\left\{x: 0<d_{\infty}(x, \mathscr{O})<r\right\}$, where $r>0$. Given an MPL system (2) in closed-loop with a feedback law $\mu(x)$, we study the stability properties of the closed-loop system:

$$
x(k)=A \otimes x(k-1) \oplus B \otimes \mu(x(k-1))
$$

The set $\mathscr{O}$ is called positive invariant for the system (8) if for all $x \in \mathscr{O}$ it follows that $A \otimes x \oplus B \otimes \mu(x) \in \mathscr{O}$.

Definition 1.2: A closed invariant set $\mathscr{O}$ is called stable in the sense of Lyapunov for the system (8) if for any $\theta>0$, there exists a $\delta>0$ such that for all $x(0)$ satisfying $d_{\infty}(x(0), \mathscr{O})<\delta$ we have $d_{\infty}(x(k), \mathscr{O})<\theta$, for all $k \geq 0$. If, furthermore, $d_{\infty}(x(k), \mathscr{O}) \rightarrow 0$ as $k \rightarrow \infty$, then $\mathscr{O}$ is asymptotically stable for $(8)$. In the case when $d_{\infty}(x(k), \mathscr{O}) \leq$ $c \gamma^{-\alpha k} d_{\infty}(x(0), \mathscr{O})$ for some $c, \alpha>0$ and $0<\gamma<1$, then the set $\mathscr{O}$ is exponentially stable.

The following theorem gives sufficient conditions for exponential stability.

Theorem 1.3: [9] The closed invariant set $\mathscr{O}$ is exponentially stable, if in a sufficient small neighborhood $\mathscr{N}(\mathscr{O}, r)$ of the set $\mathscr{O}$ there exists a functional $V$ with the following properties:

(i) $c_{1} d_{\infty}(x, \mathscr{O}) \leq V(x) \leq c_{2} d_{\infty}(x, \mathscr{O})$, for all $x \in \mathscr{N}(\mathscr{O}, r)$

(ii) $V(x(k+1))-V(x(k)) \leq-c_{3} d_{\infty}(x(k), \mathscr{O})$ for $x(0) \in$ $\mathscr{N}(\mathscr{O}, r)$, for all $k \geq 0$ provided that $x(k) \in \mathscr{N}(\mathscr{O}, r)$, where $c_{1}, c_{2}$ and $c_{3}$ are positive constants and $0<\frac{c_{3}}{c_{2}}<1$.

\section{Stabilizing RHC: CONSTRAined CASE}

The main advantage of RHC is that it can accommodate constraints on states and inputs. In this section we derive a stabilizing RHC scheme for MPL systems (5a)-(5b) where we consider constraints of the type (6), using a dual-mode approach as in [1].

\section{A. Maximal invariant set $\mathscr{O}_{\infty}$}

We consider the normalized MPL system (5a)-(5b) together with the constraints (6). We may assume that the equilibrium pair $\left(x_{\mathrm{e}}, u_{\mathrm{e}}\right)$ defined in (7) satisfies the constraints (6) (otherwise $\left(x_{\mathrm{e}}, u_{\mathrm{e}}\right)$ is determined as the optimal solution of the following linear programming problem: $\max _{u} \sum_{i} u_{i}$, s.t. $\left.x=A^{*} \otimes B \otimes u, C \otimes x \leq y_{\mathrm{t}}, H x+G u \leq h\right)$.

We consider the following closed-loop system:

$$
x(k)=A \otimes x(k-1) \oplus B \otimes u_{\mathrm{e}} .
$$

In [8] it is proved that $\mathscr{O}=\left\{x_{\mathrm{e}}\right\}$ is asymptotically stable for the closed-loop system (9). We define the state constraint set associated to the closed-loop system (9)

$$
\mathscr{O}_{0}=\left\{x \in \mathbb{R}^{n}: H x+G u_{\mathrm{e}} \leq h\right\}
$$


We define recursively for all $k \geq 1$ the sets

$$
\mathscr{O}_{k}=\left\{x \in \mathscr{O}_{0}: A \otimes x \oplus B \otimes u_{\mathrm{e}} \in \mathscr{O}_{k-1}\right\}
$$

It is trivial to see that $\mathscr{O}_{k} \subseteq \mathscr{O}_{k-1} \subseteq \cdots \subseteq \mathscr{O}_{1} \subseteq O_{0}$. Therefore, the limit of $\mathscr{O}_{k}$ exists and we have

$$
\mathscr{O}_{\infty}=\bigcap_{k \geq 0} \mathscr{O}_{k}=\lim _{k \rightarrow \infty} \mathscr{O}_{k}
$$

By induction we can prove that $x_{\mathrm{e}} \in \mathscr{O}_{k}$, for all $k \geq 0$ and therefore $x_{\mathrm{e}} \in \mathscr{O}_{\infty}$ i.e. $\mathscr{O}_{\infty}$ is non-empty.

Lemma 2.1: If Assumption $\mathbf{A}$ is satisfied then $\mathscr{O}_{k}$ is a polyhedral set having the form

$$
\mathscr{O}_{k}=\left\{x \in \mathbb{R}^{n}: H_{k} x \leq h_{k}\right\}
$$

with the matrix $H_{k} \geq 0$.

Proof: For $k=0$ the statement is obvious (see Assumption A). Let us assume that $\mathscr{O}_{k-1}=\left\{x \in \mathbb{R}^{n}: H_{k-1} x \leq\right.$ $\left.h_{k-1}\right\}$, with $H_{k-1} \geq 0$ and we prove that $\mathscr{O}_{k}$ has a similar form. Since $A \otimes x \oplus B \otimes u_{\mathrm{e}}$ is a "max" expression of the form $\left[\max _{j}\left\{a_{i j}+x_{j}, c_{i}\right\}\right]_{i}$ for some $a_{i j} \in \mathbb{R}_{\varepsilon}$ and a constant vector $c$, it is straightforward to show that the inequality $H_{k-1}\left(A \otimes x \oplus B \otimes u_{\mathrm{e}}\right) \leq h_{k-1}$ can be rewritten in the form $\bar{H}_{k} x \leq \bar{h}_{k}$, with $\bar{H}_{k} \geq 0$. Then, $H_{k}=\left[H_{k-1}^{T} \bar{H}_{k}^{T}\right]^{T} \geq 0$ and $h_{k}=\left[h_{k-1}^{T} \bar{h}_{k}^{T}\right]^{T}$.

From the previous lemma it is clear that the set $\mathscr{O}_{\infty}$ is convex (it is a countable intersection of polyhedral sets). We derive now conditions when $\mathscr{O}_{\infty}$ is a polyhedron.

Theorem 2.2: (i) If there exists a $t^{*}$ such that $\mathscr{O}_{t^{*}}=\mathscr{O}_{t^{*}+1}$ then $\mathscr{O}_{\infty}=\mathscr{O}_{t^{*}}$ (i.e. $\mathscr{O}_{\infty}$ is finitely determined and it is a polyhedral set).

(ii) The set $\mathscr{O}_{\infty}$ is the maximal positively invariant set for (9) contained in $\mathscr{O}_{0}$.

Proof: (i) Let us assume that there exists a $t^{*}$ such that $\mathscr{O}_{t^{*}}=\mathscr{O}_{t^{*}+1}$. It is obvious that $\mathscr{O}_{t^{*}+2} \subseteq \mathscr{O}_{t^{*}+1}$. Moreover, for any $x \in \mathscr{O}_{t^{*}+1}$ it follows that $A \otimes x \oplus B \otimes u_{\mathrm{e}} \in \mathscr{O}_{t^{*}}=$ $\mathscr{O}_{t^{*}+1}$, i.e. $x \in \mathscr{O}_{t^{*}+2}$. In conclusion, $\mathscr{O}_{t^{*}+1} \subseteq \mathscr{O}_{t^{*}+2}$ and thus $\mathscr{O}_{t^{*}+2}=\mathscr{O}_{t^{*}+1}=\mathscr{O}_{t^{*}}$. Iterating this procedure and using (12) we conclude that $\mathscr{O}_{\infty}=\mathscr{O}_{t^{*}}$.

(ii) Let $T \subseteq \mathscr{O}_{0}=\left\{x: H_{0} x \leq h_{0}\right\}$ be a positive invariant set for (9) and let $x \in T$. Then from the definition of a positively invariant set we have $H_{0}\left(A \otimes x \oplus B \otimes u_{\mathrm{e}}\right) \leq h_{0}$. This implies that $x \in \mathscr{O}_{1}$ (according to the recursion (11)). Therefore, $T \subseteq$ $\mathscr{O}_{1}$. By iterating this procedure we obtain that $T \subseteq \mathscr{O}_{k}$ for all $k \geq 0$. In conclusion, for any positive invariant set $T$ it follows that $T \subseteq \mathscr{O}_{\infty}$ and thus $\mathscr{O}_{\infty}$ is maximal.

From Theorem 2.2 we have obtained that if $\mathscr{O}_{\infty}$ is finitely determined then $\mathscr{O}_{\infty}$ is a polyhedron of the form $\mathscr{O}_{\infty}=\{x \in$ $\left.\mathbb{R}^{n}: H_{\infty} x \leq h_{\infty}\right\}$, where $H_{\infty} \geq 0$. Now, we give sufficient conditions under which the set $\mathscr{O}_{\infty}$ is finitely determined. Note that the recursive relation (11) can be written equivalently as

$$
\begin{array}{r}
\mathscr{O}_{k}=\left\{x \in \mathscr{O}_{k-1}: H\left(A^{\otimes^{k}} \otimes x \oplus A^{\otimes^{k-1}} \otimes B \otimes u_{\mathrm{e}} \oplus \cdots \oplus\right.\right. \\
\left.\left.B \otimes u_{\mathrm{e}}\right)+G u_{\mathrm{e}} \leq h\right\} .
\end{array}
$$

Theorem 2.3: Suppose that there exists a positive integer $t_{0}$ and $a \in \mathbb{R}^{n}$ such that $\mathscr{O}_{t_{0}} \subseteq\left\{x \in \mathbb{R}^{n}: x \leq a\right\}$. Then, $\mathscr{O}_{\infty}$ is finitely determined (i.e. $\exists t^{*}$ such that $\mathscr{O}_{\infty}=\mathscr{O}_{t^{*}}$ ).
Proof: Since $A_{i j}<0, \forall i, j$ it follows that for all $x \in \mathbb{R}^{n}$ : $A^{\otimes^{k}} \otimes x \rightarrow \varepsilon$ as $k \rightarrow \infty$. Moreover, for any $b \in \mathbb{R}^{n}$ we have: $b \oplus A \otimes b \oplus \cdots \oplus A^{\otimes^{k+n}} \otimes b=A^{*} \otimes b$, for all $k \geq 0$. Since $x_{\mathrm{e}}=A^{*} \otimes B \otimes u_{\mathrm{e}}$ is finite, there exists $t^{*} \geq \max \left\{n, t_{0}\right\}$ such that $A^{\otimes^{k}} \otimes a \leq x_{\mathrm{e}}$, for all $k \geq t^{*}$. We show that $\mathscr{O}_{t^{*}}=\mathscr{O}_{t^{*}+1}$. Since $\mathscr{O}_{t^{*}+1} \subseteq \mathscr{O}_{t^{*}}$, to complete the proof we now show that the other inclusion is also valid, i.e. $\mathscr{O}_{t^{*}} \subseteq \mathscr{O}_{t^{*}+1}$.

Let $x \in \mathscr{O}_{t^{*}} \subseteq \mathscr{O}_{t_{0}} \subseteq\left\{x \in \mathbb{R}^{n}: x \leq a\right\}$. Then, $A^{\otimes^{t^{*}+1}} \otimes x \leq$ $A^{\otimes^{t^{*}+1}} \otimes a \leq x_{\mathrm{e}}$. It follows that: $H\left(A^{\otimes^{t^{*}+1}} \otimes x \oplus A^{\otimes^{t^{*}}} \otimes B \otimes\right.$ $\left.u_{\mathrm{e}} \oplus \cdots \oplus B \otimes u_{\mathrm{e}}\right)=H\left(A^{\otimes^{t^{*}+1}} \otimes x \oplus A^{*} \otimes B \otimes u_{\mathrm{e}}\right)=H x_{\mathrm{e}} \leq$ $h-G u_{\mathrm{e}}$, i.e. $x \in \mathscr{O}_{t^{*}+1}$.

It is often the case that the set $\mathscr{O}_{0}$ can be written as $\mathscr{O}_{0}=$ $\left\{x \in \mathbb{R}_{\varepsilon}^{n}: x_{i} \leq a_{i}^{0}\right.$, for $\left.i=1, \cdots, n\right\}$, where $a_{i}^{0}$ is either a finite number or $+\infty$ (when there are no restrictions on $x_{i}$ ). Then, we can prove that all the sets $\mathscr{O}_{k}$ can be written in a similar form $\mathscr{O}_{k}=\left\{x \in \mathbb{R}_{\varepsilon}^{n}: x_{i} \leq a_{i}^{k}\right.$, for $\left.i=1, \cdots, n\right\}$, where $a_{i}^{k}$ is either a finite number or $+\infty$ (i.e. every $\mathscr{O}_{k}$ is described by at most $n$ inequalities). We prove this by induction. For $k=0$ this statement is true. Let us assume that $\mathscr{O}_{k}=\left\{x \in \mathbb{R}_{\varepsilon}^{n}: x_{i} \leq\right.$ $a_{i}^{k}$, for $\left.i=1, \cdots, n\right\}$ and we prove that $\mathscr{O}_{k+1}$ has a similar form. We denote with $a^{k}=\left[a_{1}^{k} \cdots a_{n}^{k}\right]^{T}$. From the recursive relation (11) we have:

$$
\begin{aligned}
\mathscr{O}_{k+1} & =\left\{x \in \mathbb{R}_{\varepsilon}^{n}: x \leq a^{k}, A \otimes x \leq a^{k}\right\}= \\
\left\{x \in \mathbb{R}_{\varepsilon}^{n}: x \leq a^{k}, x \leq\left(-A^{T}\right) \otimes^{\prime} a^{k}\right\} & =\left\{x \in \mathbb{R}_{\varepsilon}^{n}: x \leq a^{k+1}\right\}
\end{aligned}
$$

where $a^{k+1}=\min \left\{a^{k},\left(-A^{T}\right) \otimes^{\prime} a^{k}\right\}$. We conclude that $\mathscr{O}_{\infty}$ is described by at most $n$ inequalities and in fact $\mathscr{O}_{\infty}=\{x \in$ $\left.\mathbb{R}_{\varepsilon}^{n}: x \leq a^{\infty}\right\}$ where $a_{i}^{\infty}$ is either in $\mathbb{R}$ or equal to $+\infty$ for any $i=1, \cdots, n$.

Note that the results obtained in this section concerning the maximal positively invariant set $\mathscr{O}_{\infty}$ for the MPL system (9) are similar to the one obtained in [12] for the linear case.

\section{B. Stable constrained RHC}

In this section it is assumed that the maximal positively invariant set $\mathscr{O}_{\infty}=\left\{x \in \mathbb{R}^{n}: H_{\infty} x \leq h_{\infty}\right\}$ is available, where $H_{\infty} \geq 0$. We give now a lemma that will be used in the sequel:

Lemma 2.4: (i) Let $X_{\mathrm{f}}=\left\{x \in \mathbb{R}^{n}: P x \leq q\right\}$, where $P \geq 0$. Then,

$$
d_{\infty}\left(x_{0}, X_{\mathrm{f}}\right)=\min _{x \in X_{\mathrm{f}}} \max \left\{\left\|x_{0}-x\right\|_{\oplus}, 0\right\}
$$

(ii) In particular if $X_{\mathrm{f}}(\alpha):=\left\{x \in \mathbb{R}^{n}: x \leq \alpha\right\}$ then

$$
d_{\infty}\left(x_{0}, X_{\mathrm{f}}(\alpha)\right)=\max \left\{\left\|x_{0}-\alpha\right\|_{\oplus}, 0\right\}
$$

Proof: (i) It is straightforward to see that the statement is true when $x_{0} \in X_{\mathrm{f}}$. Therefore, we consider the case when $x_{0} \notin X_{\mathrm{f}}$, i.e. $d_{\infty}\left(x_{0}, X_{\mathrm{f}}\right)>0$. We prove this case by contradiction. Let $x^{*} \in X_{\mathrm{f}}$ be the optimal solution, i.e. $0<d_{\infty}\left(x_{0}, X_{\mathrm{f}}\right)=\left\|x_{0}-x^{*}\right\|_{\infty}$. We define the set $\mathscr{I} \subseteq \underline{n}$ as follows: if $i \in \mathscr{I}$ then $\left\|x_{0}-x^{*}\right\|_{\infty}=x_{i}^{*}-\left(x_{0}\right)_{i}>0$ and for any $j \in \underline{n} \backslash \mathscr{I}:\left\|x_{0}-x^{*}\right\|_{\infty}>\left(x_{0}\right)_{j}-x_{j}^{*}$; otherwise, if such $\mathscr{I}$ does not exists, then define $\mathscr{I}=\emptyset$.

Assume that $\mathscr{I} \neq \emptyset$. Then, we define $x_{\text {feas }}$ as: $\left(x_{\text {feas }}\right)_{i}=$ $\left(x_{0}\right)_{i}$, if $i \in \mathscr{I}$ and $\left(x_{\text {feas }}\right)_{i}=x_{i}^{*}$, if $i \notin \mathscr{I}$. Since $P \geq 0$ and $x_{\text {feas }} \leq x^{*}, x_{\text {feas }} \neq x^{*}$ it follows that $x_{\text {feas }} \in X_{\mathrm{f}}$. Moreover, $0<\bar{d}_{\infty}\left(x_{0}, X_{\mathrm{f}}\right)=\left\|x_{0}-x^{*}\right\|_{\infty}=\max _{i \in \underline{n}}\left\{x_{i}^{*}-\left(x_{0}\right)_{i},\left(x_{0}\right)_{i}-\right.$ 
$\left.x_{i}^{*}\right\} \leq\left\|x_{\text {feas }}-x_{0}\right\|_{\infty}=\max _{i \notin \mathscr{I}}\left\{x_{i}^{*}-\left(x_{0}\right)_{i},\left(x_{0}\right)_{i}-x_{i}^{*}, 0\right\}<$ $\max _{i \in \underline{n}}\left\{x_{i}^{*}-\left(x_{0}\right)_{i},\left(x_{0}\right)_{i}-x_{i}^{*}\right\}=\left\|x_{0}-x^{*}\right\|_{\infty}$ i.e. a contradiction. Therefore, $\mathscr{I}=\emptyset$ and then $\left\|x_{0}-x^{*}\right\|_{\infty}=\left\|x_{0}-x^{*}\right\|_{\oplus}$.

(ii) If $x_{0} \notin X_{\mathrm{f}}(\alpha)$ and $x \leq \alpha$, the following inequality is valid: $\max _{i \in \underline{n}}\left\{\left(x_{0}\right)_{i}-x_{i}\right\} \geq \max _{i \in \underline{n}}\left\{\left(x_{0}\right)_{i}-\alpha_{i}\right\}$. We conclude that $\min _{x \in X_{\mathrm{f}}(\alpha)} \max _{i \in \underline{n}}\left\{\left(x_{0}\right)_{i}-x_{i}\right\} \geq \max _{i \in \underline{n}}\left\{\left(x_{0}\right)_{i}-\right.$ $\alpha_{i}$. From (i) it follows that $d_{\infty}\left(x_{0}, X_{\mathrm{f}}(\alpha)\right) \geq \max _{i \in \underline{n}}\left\{\left(x_{0}\right)_{i}-\right.$ $\left.\alpha_{i}\right\}=\left\|x_{0}-\alpha\right\|_{\oplus}$ (according to the first part of this lemma). But $d_{\infty}\left(x_{0}, X_{\mathrm{f}}(\alpha)\right) \leq\left\|x_{0}-\alpha\right\|_{\oplus}$ since $\alpha \in X_{\mathrm{f}}(\alpha)$. It follows that $d_{\infty}\left(x_{0}, X_{\mathrm{f}}(\alpha)\right)=\left\|x_{0}-\alpha\right\|_{\oplus}$.

For initial conditions $x(0), u(0)$ and a future input sequence $\tilde{u}=(u(1) \cdots u(N))$, the following cost function is introduced:

$$
J(x(0), \tilde{u})=\sum_{j=0}^{N} d_{\infty}\left(x(j), \mathscr{O}_{\infty}\right)+\beta\left\|u(j)-u_{\mathrm{e}}\right\|_{\infty},
$$

where $\beta>0$ and $N$ is the prediction horizon. Usually, it is the case that $\mathscr{O}_{\infty}=\left\{x: x \leq a_{\infty}\right\}$. Then, from Lemma 2.4 we have that $J(x(0), \tilde{u})=\sum_{j=0}^{N} \max \left\{\left\|x(j)-a_{\infty}\right\|_{\oplus}, 0\right\}+$ $\beta\left\|u(j)-u_{\mathrm{e}}\right\|_{\infty}$. In the context of manufacturing systems the first term expresses the tardiness with respect to $a_{\infty}$, while the second term penalizes the delay with respect to $u_{\mathrm{e}}$.

Since we want to feed raw material as late as possible, we impose the constraint $u(k) \geq u_{\mathrm{e}}$ for all $k \geq 1$. For simplicity, we assume that $B \otimes u_{\mathrm{e}}$ is a finite vector. We have that $x(k) \geq$ $B \otimes u_{\mathrm{e}}$ for all $k \geq 1$. In conclusion, $\mathscr{O}_{\infty} \cap\left\{x: x \geq B \otimes u_{\mathrm{e}}\right\}$, which is bounded, is in fact an invariant set for (9). Given $x(k-1)$ and $u(k-1)$, the RHC optimization problem at stage $k-1$ is defined as follows:

$$
\begin{aligned}
& J^{*}(x(k-1))=\min _{\tilde{u}(k)} J(x(k-1), \tilde{u}(k)) \\
& \text { s.t. }\left\{\begin{array}{l}
u(k+j \mid k-1)-u(k+j-1 \mid k-1) \geq-\rho \\
u(k+j \mid k-1) \geq u_{\mathrm{e}} \\
H x(k+j \mid k-1)+G u(k+j \mid k-1) \leq h, \forall j \in\{0, \cdots, N-1\} \\
u_{\mathrm{e}}-u(k+N-1 \mid k-1) \geq-\rho \\
x(k+N-1 \mid k-1) \in \mathscr{O}_{\infty}
\end{array}\right.
\end{aligned}
$$

where $x(k+j \mid k-1)$ is the system state at $k+j$ as predicted at $k-1$, based on (5a)-(5b), $x(k-1 \mid k-1)=x(k-$ $1), u(k-1 \mid k-1)=u(k-1)$ and the future input sequence $\tilde{u}(k):=(u(k \mid k-1) \cdots u(k+N-1 \mid k-1))$. By including extra variables and using Lemma 2.4, the entire optimization problem can be written as a linear program. We apply the optimal controller in a receding horizon fashion: at event $k$ we apply $u^{\mathrm{RHC}}(k):=u^{*}(k \mid k-1)$ to the system (5a)-(5b), where $\tilde{u}^{*}(k)$ is the optimal solution of (15). Recall that the set $\mathscr{O}_{\infty} \cap\left\{x: x \geq B \otimes u_{\mathrm{e}}\right\}$ is bounded. We can derive the following lemma:

Lemma 2.5: There exist $r>0$ and $c_{2}>1$ such that for all $x \in \mathscr{N}\left(\mathscr{O}_{\infty}, r\right)$ we have

$$
d_{\infty}\left(x, \mathscr{O}_{\infty}\right) \leq J^{*}(x) \leq c_{2} d_{\infty}\left(x, \mathscr{O}_{\infty}\right)
$$

Proof: Let us take $r>0$. The following facts are easy to prove.

Fact 1: For any finite vectors $x, u, y, v$ and matrices $A, B$ satisfying Assumption 1 we have:

$\|A \otimes x \oplus B \otimes u-A \otimes y \oplus B \otimes v\|_{\infty} \leq\|x-y\|_{\infty} \oplus\|u-v\|_{\infty}$
Fact 2: It is well-known (see [13], [14]) that the optimal RHC solution of (15) is a piecewise affine function of the current state $x(k-1): u^{*}(k+j \mid k-1)=\mu(x(k-1))$, for all $j \in\{0, \cdots, N-1\}$, where $\mu(\cdot)$ is a piecewise affine function.

Fact 3: Given a polytope $\mathscr{P} \subseteq \mathscr{N}\left(\mathscr{O}_{\infty}, r\right) \cap\left\{x: x \geq B \otimes u_{\mathrm{e}}\right\}$ then, exists a $c>0$ such that $\|F x+g\|_{\infty} \leq c d_{\infty}\left(x, \mathscr{O}_{\infty}\right), \forall x \in$ $\mathscr{P}$ for any matrix $F$ and vector $g$. (Indeed, the functions $x \longmapsto\|F x+g\|_{\infty}$ and $x \longmapsto d_{\infty}\left(x, \mathscr{O}_{\infty}\right)$ are continuous on the compact set $\mathscr{P}$. Moreover, $d_{\infty}\left(x, \mathscr{O}_{\infty}\right)>0$ for all $x \in$ $\mathscr{P}$. Then, the function $x \longmapsto \frac{\|F x+g\|_{\infty}}{d_{\infty}\left(x, \mathscr{O}_{\infty}\right)}$ is continuous on the compact set $\mathscr{P}$. From the Weierstrass theorem, this function is bounded. Therefore, there exists a $c>0$ such that $\frac{\|F x+g\|_{\infty}}{d_{\infty}\left(x, \mathscr{O}_{\infty}\right)} \leq c$ for all $x \in \mathscr{P}$.)

Using Fact 2 and Fact 3 we conclude that $\| u^{*}(k+j \mid k-$ $1)-u_{\mathrm{e}} \|_{\infty} \leq \bar{c}_{j} d_{\infty}\left(x(k-1), \mathscr{O}_{\infty}\right)$ for all $x(k-1) \in \mathscr{N}\left(\mathscr{O}_{\infty}, r\right)$, where $\bar{c}_{j}>0$.

From Fact 1 and Fact 3 we have

$$
\begin{aligned}
& d_{\infty}\left(x(k+j \mid k-1), \mathscr{O}_{\infty}\right)=\min _{x \in \mathscr{O}_{\infty}} \| A^{\otimes^{j+1}} \otimes x(k-1) \oplus \\
& A^{\otimes^{j}} \otimes B \otimes u^{*}(k \mid k-1) \oplus \cdots \oplus B \otimes u^{*}(k+j \mid k-1)-x \|_{\infty} \leq \\
& \min _{x \in \mathscr{O}_{\infty}} \| A^{\otimes^{j+1}} \otimes x(k-1) \oplus A^{\otimes^{j}} \otimes B \otimes u^{*}(k \mid k-1) \oplus \cdots \oplus \\
& B \otimes u^{*}(k+j \mid k-1)-A^{\otimes^{j+1}} \otimes x \oplus A^{\otimes^{j}} \otimes B \otimes u_{\mathrm{e}} \oplus \cdots \oplus \\
& B \otimes u_{\mathrm{e}} \|_{\infty} \leq d_{\infty}\left(x(k-1), \mathscr{O}_{\infty}\right) \oplus \bar{c}_{j} d_{\infty}\left(x(k-1), \mathscr{O}_{\infty}\right) \oplus \cdots \oplus \\
& \bar{c}_{1} d_{\infty}\left(x(k-1), \mathscr{O}_{\infty}\right) \leq \tilde{c}_{j} d_{\infty}\left(x(k-1), \mathscr{O}_{\infty}\right), \forall j \in\{0, \cdots, N-1\}
\end{aligned}
$$

where $\tilde{c}_{j}>0$. In conclusion, there exists a $c_{2}>1$ such that $J^{*}(x(k-1)) \leq c_{2} d_{\infty}\left(x(k-1), \mathscr{O}_{\infty}\right)$ for all $x(k-1) \in$ $\mathscr{N}\left(\mathscr{O}_{\infty}, r\right)$. It is obvious that $J^{*}(x(k-1)) \geq d_{\infty}\left(x(k-1), \mathscr{O}_{\infty}\right)$ for all $x(k-1) \in N\left(\mathscr{O}_{\infty}, r\right)$.

We define the feasible set:

$$
X_{N}^{\mathrm{RHC}}=\left\{x \in \mathbb{R}_{\varepsilon}^{n}:(15) \text { is feasible for } x(k-1)=x\right\}
$$

Theorem 2.6: If $x(0) \in X_{N}^{\mathrm{RHC}}$ then all subsequent stages of the optimization problem (15) will be feasible. Moreover, the bounded set $\mathscr{O}_{\infty} \cap\left\{x: x \geq B \otimes u_{\mathrm{e}}\right\}$ is exponentially stable for the system (5a)-(5b) in closed-loop with the RHC controller $u^{\mathrm{RHC}}(k)=u^{*}(k \mid k-1)$.

Proof: The proof is done by induction. If (15) has an optimal solution at step $k-1: \tilde{u}^{*}(k)=\left(u^{*}(k \mid k-1) \cdots u^{*}(k+\right.$ $N-1 \mid k-1))$, then at step $k$ a feasible solution is $u_{\text {feas }}=$ $\left(u^{*}(k+1 \mid k-1) \cdots u^{*}(k+N-1 \mid k-1) u_{\mathrm{e}}\right)$ since $\mathscr{O}_{\infty}$ is a positively invariant set, $x(k+N-1 \mid k-1) \in \mathscr{O}_{\infty}$ and then $u_{\mathrm{e}}$ keeps the state in $\mathscr{O}_{\infty}$. Using the receding horizon principle the state at $k$ becomes $x(k)=A \otimes x(k-1) \oplus B \otimes u^{\mathrm{RHC}}(k)=$ $x(k \mid k-1)$.

$$
\begin{aligned}
& \text { Since } x(k+N-1 \mid k-1) \in \mathscr{O}_{\infty}, \text { we have } \\
& \begin{aligned}
J^{*}(x(k))-J^{*}(x(k-1)) \leq & J\left(x(k), u_{\text {feas }}\right)-J^{*}(x(k-1)) \leq \\
& -d_{\infty}\left(x(k-1), \mathscr{O}_{\infty}\right)
\end{aligned}
\end{aligned}
$$

Therefore, $\left\{J^{*}(x(k))\right\}_{k \geq 0}$ is a non-increasing sequence and from Lemma 2.5 we have

$$
d_{\infty}\left(x(k-1), \mathscr{O}_{\infty}\right) \leq J^{*}(x(k-1)) \leq c_{2} d_{\infty}\left(x(k-1), \mathscr{O}_{\infty}\right)
$$

Using Theorem 1.3 and $x(k) \geq B \otimes u_{\mathrm{e}}$ for all $k \geq 1$, we conclude that the compact set $\mathscr{O}_{\infty} \cap\left\{x: x \geq B \otimes u_{\mathrm{e}}\right\}$ is 
exponentially stable for the system (5a) in closed-loop with the RHC controller provided by (15).

Assuming $x(k) \in \mathscr{O}_{\infty}$ we switch then to the feasible controller $u_{\mathrm{e}}$ for all the subsequent motion and we need finite number of steps to attain $x_{\mathrm{e}}$. Indeed, $x(k+j)=A^{\otimes^{j}} \otimes x(k) \oplus$ $\left(\bigoplus_{i=1}^{j} A^{\otimes^{j-i}} \otimes B \otimes u_{\mathrm{e}}\right) \in \mathscr{O}_{\infty}$ for any $j \geq 1$. Since $A^{\otimes^{j}} \rightarrow \boldsymbol{E}$ the first term $A^{\otimes^{j}} \otimes x(k) \rightarrow \mathcal{E}$ while the second is equal to $x_{\mathrm{e}}$ for $j \geq n$.

Interpretation of Lyapunov stability: In the context of discrete-event systems, the Lyapunov stability of the compact set $\mathscr{O}_{\infty} \cap\left\{x: x \geq B \otimes u_{\mathrm{e}}\right\}$ implies boundedness of the buffer levels.

\section{TIME-OPTIMAL CONTROL}

Given a maximum horizon length $N_{\max }$ we now consider the problem of ensuring that the completion times after $N$ events, where $N \in\left\{1,2, \cdots, N_{\max }\right\}$ are less than or equal to a specified target time $\alpha(x(N) \leq \alpha$ with the initial conditions $x(0)$ and $u(0)$ ), using the largest controller that satisfies the state-input constraints (6). Note that such a problem, but without considering input and state constraints, was considered also in [4] in terms of lattice theory.

We define an equivalent system for $(5 a)-(5 b)$ such that we do not need to impose the constraint $u(k+1)-u(k) \geq-\rho$, this constraint being satisfied automatically. We introduce a new state vector $x_{\text {new }}(k)=\left[\begin{array}{ll}x^{T}(k) & u_{\text {new }}^{T}(k)\end{array}\right]^{T}$ with the dynamics:

$$
\left\{\begin{array}{l}
x_{\text {new }}(k)=\left[\begin{array}{cc}
A & B-\rho \\
\mathcal{E} & E_{m}-\rho
\end{array}\right] \otimes x_{\text {new }}(k-1) \oplus\left[\begin{array}{c}
B \\
E_{m}
\end{array}\right] \otimes u(k) \\
y_{\text {new }}(k)=\left[\begin{array}{ll}
C & \boldsymbol{E}
\end{array}\right] \otimes x_{\text {new }}(k)
\end{array}\right.
$$

and the extra constraint:

$$
u_{\text {new }}(k) \leq u(k)
$$

We denote with $A_{\text {new }}=\left[\begin{array}{cc}A & B-\rho \\ \mathcal{E} & E_{m}-\rho\end{array}\right], B_{\text {new }}=\left[\begin{array}{c}B \\ E_{m}\end{array}\right]$ and $C_{\text {new }}=\left[\begin{array}{ll}C & \boldsymbol{\varepsilon}\end{array}\right]$. Given the initial conditions $x(0)$ and $u(0)$ for the system (5a)-(5b) with constraints (6) and the initial conditions $x_{\text {new }}(0)=\left[x(0)^{T} u(0)^{T}\right]^{T}$ and $u(0)$ for the new system (16) with the extra constraint (17) then by applying the same input $u(k)$ for both systems we obtain that the first $n$ components of $x_{\text {new }}(k)$ coincide with $x(k)$ and the last $m$ components of $x_{\text {new }}(k)$ coincide with $u(k)$. Note that the constraints (6) for the normalized system (5a)- (5b) can be written for the new system (16) as $\left[\begin{array}{ll}H & 0\end{array}\right] x_{\text {new }}(k)+G u(k) \leq$ $h, \quad\left[\begin{array}{ll}\mathbf{0} & I_{m}\end{array}\right] x_{\text {new }}(k)-I_{m} u(k) \leq 0$, i.e.

$$
H_{\text {new }} x_{\text {new }}(k)+G_{\text {new }} u(k) \leq h_{\text {new }}
$$

where $H_{\text {new }} \geq 0$. Moreover, for the new system (16) the target time is $\alpha_{\text {new }}=\left[\alpha^{T}\left(\left(-B^{T}\right) \otimes^{\prime} \alpha\right)^{T}\right]^{T}$. The time-optimal control problem can be posed in terms of an optimization

$$
{ }^{4} \text { Here } H_{\text {new }}=\left[\begin{array}{cc}
H & \mathbf{0} \\
\mathbf{0} & I_{m}
\end{array}\right], G_{\text {new }}=\left[\begin{array}{c}
G \\
-I_{m}
\end{array}\right] \text { and } h_{\text {new }}=\left[\begin{array}{c}
h \\
0
\end{array}\right] .
$$

problem: given $x_{\text {new }}:=x_{\text {new }}(0)$, find

$$
\left\{\begin{array}{l}
N^{0}\left(x_{\text {new }}\right)=\max _{(N, u(1), \cdots, u(N))} N \\
\text { s.t. } x_{\text {new }}(j)=A_{\text {new }} \otimes x_{\text {new }}(j-1) \oplus B_{\text {new }} \otimes u(j) \\
\\
H_{\text {new }} x_{\text {new }}(j)+G_{\text {new }} u(j) \leq h_{\text {new }}, \forall j \in \underline{N} \\
\quad x_{\text {new }}(N) \leq \alpha_{\text {new }}
\end{array}\right.
$$

making $u(1), \cdots, u(N)$ as big as possible. We denote with $\tilde{X}_{N}$ the set of initial states such that after $N$ steps the trajectory is below $\alpha$ applying the largest controller:

$$
\tilde{X}_{N}=\left\{x_{\text {new }}: x_{\text {new }}(N) \leq \alpha_{\text {new }}\right\}
$$

We give first a lemma that will be useful in the sequel:

Lemma 3.1: [15] Suppose $Z=\{(x, u): \bar{H} x+\bar{G} u \leq \bar{h}\}$, with $\bar{H} \geq 0$. Let $X$ be defined as $X=\left\{x \in \mathbb{R}^{n}: \exists u\right.$ s.t. $(x, u) \in$ $Z\}$. Then, $X=\{x: \tilde{H} x \leq \tilde{h}\}$, where $\tilde{H} \geq 0$.

We determine the expression of $\tilde{X}_{N}$ using dynamic programming. We initialize with $\tilde{X}_{0}=\left\{x_{\text {new }}: x_{\text {new }} \leq \alpha_{\text {new }}\right\}$. The set $\tilde{X}_{1}$ is defined as follows:

$$
\begin{aligned}
\tilde{X}_{1}=\left\{x_{\text {new }}: \exists u \text { s.t. } A_{\text {new }} \otimes x_{\text {new }} \oplus B_{\text {new }} \otimes u \in \tilde{X}_{0},\right. \\
\left.H_{\text {new }}\left(A_{\text {new }} \otimes x_{\text {new }} \oplus B_{\text {new }} \otimes u\right)+G_{\text {new }} u \leq h_{\text {new }}\right\} \\
=\left\{x_{\text {new }}: \exists u \text { s.t. } \bar{H}_{1} x_{\text {new }}+\bar{G}_{1} u \leq \bar{h}_{1}\right\}
\end{aligned}
$$

for some matrices $\bar{H}_{1}, \bar{G}_{1}$ and $\bar{h}_{1}$, with $\bar{H}_{1} \geq 0$. Using Lemma 3.1 we conclude: $\tilde{X}_{1}=\left\{x_{\text {new }}: \tilde{H}_{1} x_{\text {new }} \leq \tilde{h}_{1}\right\}$, with $\tilde{H}_{1} \geq 0$. Moreover, we search for the largest controller $u$. In order to find a Pareto optimal $u$ we use the following criterion:

$$
\max _{u} \sum_{i=1}^{m} u_{i} \text { s.t. } \bar{H}_{1} x_{\text {new }}+\bar{G}_{1} u \leq \bar{h}_{1}
$$

Solving this optimization problem as a parametric linear program with the parameter $x_{\text {new }}$ we find the time-optimal controller $u_{1}^{\mathrm{t}}(\cdot): \tilde{X}_{1} \rightarrow \mathbb{R}^{m}$ which is a continuous piecewise affine function of the state $x_{\text {new }}$.

Iterating this procedure backwards, we can compute

$$
\begin{aligned}
\tilde{X}_{N}=\left\{x_{\text {new }}: \exists u \text { s.t. } A_{\text {new }} \otimes x_{\text {new }} \oplus B_{\text {new }} \otimes u \in \tilde{X}_{N-1},\right. \\
\left.H_{\text {new }}\left(A_{\text {new }} \otimes x_{\text {new }} \oplus B_{\text {new }} \otimes u\right)+G_{\text {new }} u \leq h_{\text {new }}\right\} \\
=\left\{x_{\text {new }}: \exists u \text { s.t. } \bar{H}_{N} x_{\text {new }}+\bar{G}_{N} u \leq \bar{h}_{N}\right\}
\end{aligned}
$$

with matrix $\bar{H}_{N}$ having all entries non-negative. Using Lemma (3.1) we obtain that $\tilde{X}_{N}=\left\{x_{\text {new }}: \tilde{H}_{N} x_{\text {new }} \leq \tilde{h}_{N}\right\}$, with $\tilde{H}_{N} \geq 0$.

Similarly, we obtain the piecewise affine time-optimal controller $u_{N}^{\mathrm{t}}(\cdot): \tilde{X}_{N} \rightarrow \mathbb{R}^{m}$ by solving a parametric linear program in $x_{\text {new }}$ :

$$
\max _{u} \sum_{j=1}^{m} u_{j} \text { s.t. } \bar{H}_{N} x_{\text {new }}+\bar{G}_{N} u \leq \bar{h}_{N} .
$$

It follows that

$$
N^{0}\left(x_{\text {new }}\right)=\max \left\{N \in \underline{N_{\text {max }}}: x_{\text {new }} \in \tilde{X}_{N}\right\} .
$$

The time-optimal controller is implemented as follows:

1) For each $N \in N_{\max }$, find $\tilde{X}_{N}$. Define $N:=N^{0}\left(x_{\text {new }}(0)\right)$.

2) Apply the control policy $u(k)=u_{N-k+1}^{\mathrm{t}}\left(x_{\text {new }}(k-1)\right)$ for $k=1,2, \cdots, N^{0}\left(x_{\text {new }}(0)\right)$. 


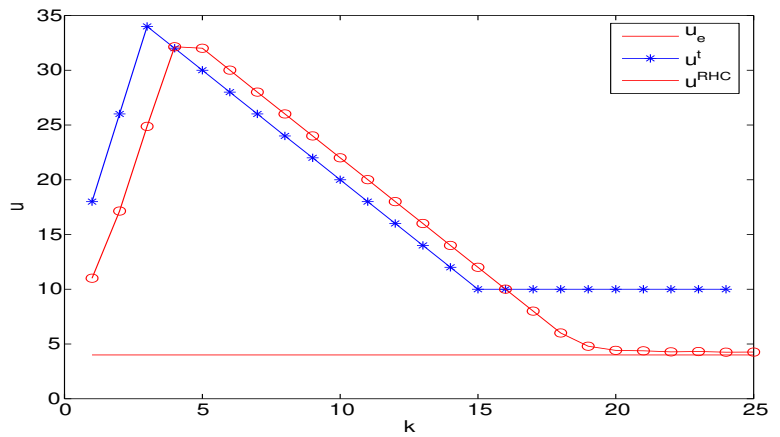

Fig. 1. The RHC controller and the time-optimal controller.

\section{EXAMPLE}

We consider the following system:

$x_{\mathrm{sys}}(k)=\left[\begin{array}{cccc}\varepsilon & 1 & \varepsilon & \varepsilon \\ \varepsilon & \varepsilon & 2 & \varepsilon \\ \varepsilon & \varepsilon & \varepsilon & 3 \\ 4 & \varepsilon & \varepsilon & \varepsilon\end{array}\right] \otimes x_{\mathrm{sys}}(k-1) \oplus\left[\begin{array}{c}2 \\ \varepsilon \\ \varepsilon \\ \varepsilon\end{array}\right] \otimes u_{\mathrm{sys}}(k)$

For this example the MPA eigenvalue is $\lambda=2.5$. We consider the reference signal $r_{\text {sys }}(k)=\left[\begin{array}{llll}17 & 15 & 1 & 10\end{array}\right]^{T}+4.5 k$. We take the following constraints:

$$
\left\{\begin{array}{l}
u_{\mathrm{sys}}(k)-u_{\mathrm{sys}}(k+1) \leq 0 \\
x_{\mathrm{sys}, 1}(k)-u_{\mathrm{sys}}(k) \leq 4 \\
x_{\mathrm{sys}, 2}(k)-x_{\mathrm{sys}, 1}(k) \leq 9
\end{array}\right.
$$

Note that the constraint $x_{\mathrm{sys}, 2}(k)-x_{\mathrm{sys}, 1}(k) \leq 9$ is implied by the more conservative constraint $x_{\mathrm{sys}, 2}(k)-u_{\text {sys }}(k) \leq 11$.

We obtain for the normalized system the invariant set

$$
\mathscr{O}_{\infty}=\mathscr{O}_{3}=\left\{x \in \mathbb{R}_{\varepsilon}^{n}:\left[\begin{array}{llll}
1 & 0 & 0 & 0 \\
0 & 1 & 0 & 0 \\
0 & 0 & 1 & 0 \\
0 & 0 & 0 & 1
\end{array}\right] x \leq\left[\begin{array}{r}
5 \\
7 \\
9 \\
11
\end{array}\right]\right\}
$$

The initial conditions are $x(0)=\left[\begin{array}{llll}20 & 30 & 40 & 50\end{array}\right]^{T}, u(0)=$ 20. The plots correspond to the normalized system. Fig. 1 displays both: the RHC controller and the time optimal controller while the constraints for the RHC controller are depicted in Fig. 2. Note that the RHC controller keeps the system behavior as close as possible to the constraints.

\section{Conclusions}

In this paper we have discussed the problem of stabilization of an MPL system using an RHC approach. We have considered state-input constraints and using a dualmode RHC scheme we have proved that the system is exponentially stable in the sense of Lyapunov in the closedloop with the RHC controller. Moreover the optimization problem that is solved at each step is a linear program for which efficient algorithms exist. We have also derived a time-optimal controller that satisfies the constraints using parametric linear programming.

Acknowledgments: Research supported by the STW projects "Model predictive control for hybrid systems" (DMR.5675), "Multiagent control of large-scale hybrid systems" (DWV.6188) and
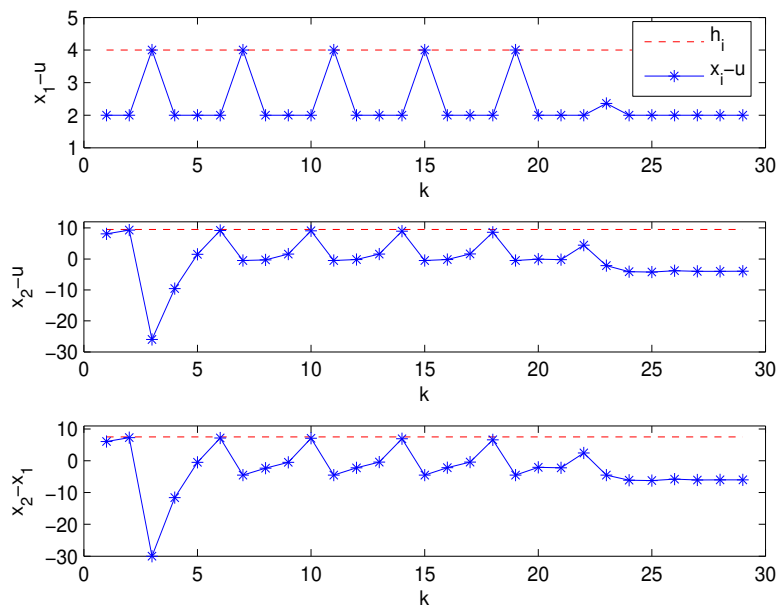

Fig. 2. Constraints. Sometimes the constraints are active.

HYCON "HYbrid CONtrol: Taming Heterogeneity and Complexity of Networked Embedded Systems (HYCON)" (FP6-IST-511368).

\section{REFERENCES}

[1] D. Mayne, J. Rawlings, C. Rao, and P. Scokaert, "Constrained model predictive control: Stability and optimality," Automatica, vol. 36, pp. 789-814, 2000.

[2] J. Maciejowski, Predictive Control with Constraints. Harlow, England: Prentice Hall, 2002.

[3] B. De Schutter and T. van den Boom, "Model predictive control for max-plus-linear discrete event systems," Automatica, vol. 37, no. 7, pp. 1049-1056, July 2001.

[4] F. Baccelli, G. Cohen, G. Olsder, and J. Quadrat, Synchronization and Linearity. New York: John Wiley \& Sons, 1992.

[5] J. Boimond and J. Ferrier, "Internal model control and max-algebra: Controller design," IEEE Transactions on Automatic Control, vol. 41, no. 3, pp. 457-461, Mar. 1996.

[6] E. Menguy, J. Boimond, L. Hardouin, and J. Ferrier, "A first step towards adaptive control for linear systems in max algebra," Discrete Event Dynamic Systems: Theory and Applications, vol. 10, no. 4, pp. 347-367, 2000.

[7] D. Cofer and V. Garg, "Supervisory control of real-time discreteevent systems using lattice theory," IEEE Transactions on Automatic Control, vol. 41, no. 2, pp. 199-209, Feb. 1996.

[8] I. Necoara, T. van den Boom, B. De Schutter, and J. Hellendoorn, "Stabilization of MPL systems using model predictive control: The unconstrained case," Delft Center for Systems and Control, Delft University of Technology, Delft, The Netherlands, Tech. Rep. 06-006, January 2006, submitted to a journal.

[9] K. Passino and K. Burgess, Stability Analysis of Discrete Event Systems. New York: John Wiley \& Sons, 1998.

[10] P. Butkovic, "Necessary solvability conditions of systems of linear extremal equations," Discrete Applied Mathematics, vol. 10, pp. 1926, 1985.

[11] J. Mairesse, "A graphical approach of the spectral theory in the max-plus algebra," IEEE Transactions on Automatic Control, vol. 40, no. 10 , pp. 1783-1789, Oct. 1995.

[12] E. Gilbert and K. T. Tan, "Linear systems with state and control constraints:the theory and applications of maximal output admissible sets," IEEE Transactions on Automatic Control, vol. 36, no. 9, pp. 1008-1020, September 1991.

[13] E. Kerrigan and D. Mayne, "Optimal control of constrained, piecewise affine systems with bounded disturbances," in Proceedings of the 2002 IEEE Conference on Decision and Control, Las Vegas, Nevada, Dec. 2002.

[14] F. Borelli, A. Bemporad, and M. Morari, "A geometric algorithm for multi-parametric linear programming," Journal of Optimization Theory and Applications, vol. 118, no. 3, pp. 515-540, Sept. 2003.

[15] I. Necoara, E. Kerrigan, B. De Schutter, and T. van den Boom, "Finitehorizon min-max control of max-plus-linear systems," Delft Center for Systems and Control, Delft University of Technology, Delft, The Netherlands, Tech. Rep. 06-002, Dec. 2005, submitted to a journal. 